\title{
Model for Infrared Properties of Extremely Young Galaxies
}

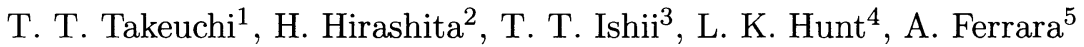

${ }^{1}$ NAOJ, Tokyo, Japan

${ }^{2}$ Nagoya U., Nagoya, Japan

${ }^{3}$ Kyoto U., Kyoto, Japan

${ }^{4}$ CAISMI-IRA/CNR, Firenze, Italy

${ }^{5}$ SISSA, Trieste, Italy

\begin{abstract}
We constructed a model of the infrared spectral energy distribution for very young galaxies by taking into account the dust size distribution in the early stage of galaxy evolution.
\end{abstract}

\section{Dust Emission from Very Young Galaxies}

We have developed a model for the evolution of dust content and the IR SED of extremely young galaxies based on Hirashita et al. (2002) with the size distribution by Todini \& Ferrara (2001). We found that, small silicate grains are subject to stochastic heating even in a strong UV field, resulting in a broad temperature distribution and strong MIR continuum emission. Larger carbon grains are in thermal equilibrium at $T \simeq 50-100 \mathrm{~K}$, and they also contribute to the MIR. We present the evolution of SEDs and IR extinction of very young, low-metallicity galaxies in Fig. 1.

\section{SED of Local Low Metallicity Dwarf Galaxies}

While high- $z$ primeval galaxies are still beyond the ability of present experiments, local starbursts are often assumed to be good representatives of the star forming activity at high redshifts. We successfully applied the model to the IR SED of SBS 0335-052, a low metallicity $\left(1 / 41 Z_{\odot}\right)$ dwarf galaxy with an unusually strong MIR flux (Fig. 2: left panel). We found the SED, optical properties and extinction of the star forming region to be consistent with a very young (age $\left.\simeq 6.5 \times 10^{6} \mathrm{yr}\right)$ and compact (radius $\simeq 20 \mathrm{pc}$ ) starburst. We also predict the SED of another extremely low-metallicity galaxy, I Zw 18, for future observational tests (Fig. 2: right panel). We estimate the FIR luminosity of I $\mathrm{Zw} 18$ to be as low as $L_{\text {FIR }} \sim 10^{7-7.5} L_{\odot}$, depending on the uncertainty of dust mass. Details of this work can be found in Takeuchi et al. (2003).

\section{References}

Hirashita, H., Hunt, L. K., \& Ferrara, A. 2002, MNRAS, 330, L19 
Takeuchi, T. T., Hirashita, H., Ishii, T. T., Hunt, L. K., \& Ferrara, A. 2003, MNRAS, 343, 839

Todini, P., \& Ferrara, A. 2001, MNRAS, 325, 726
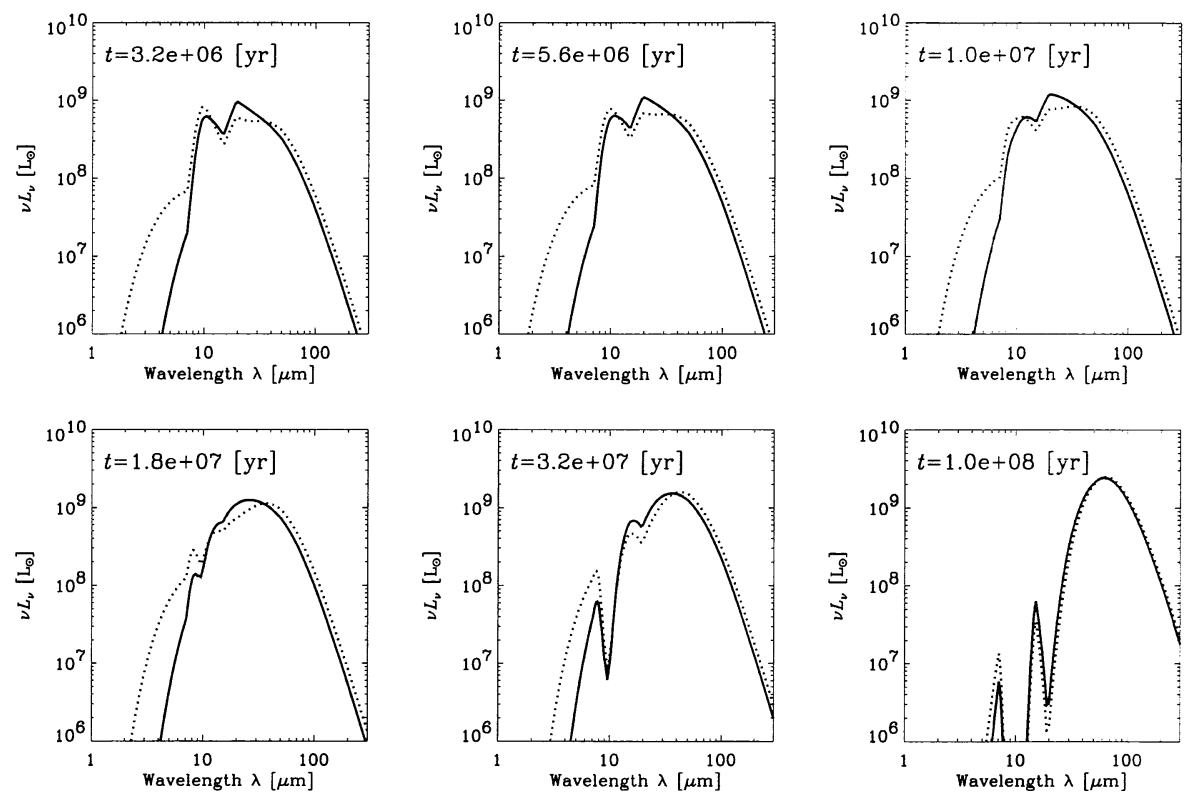

Figure 1. An example of an IR SED of a very young galaxy.
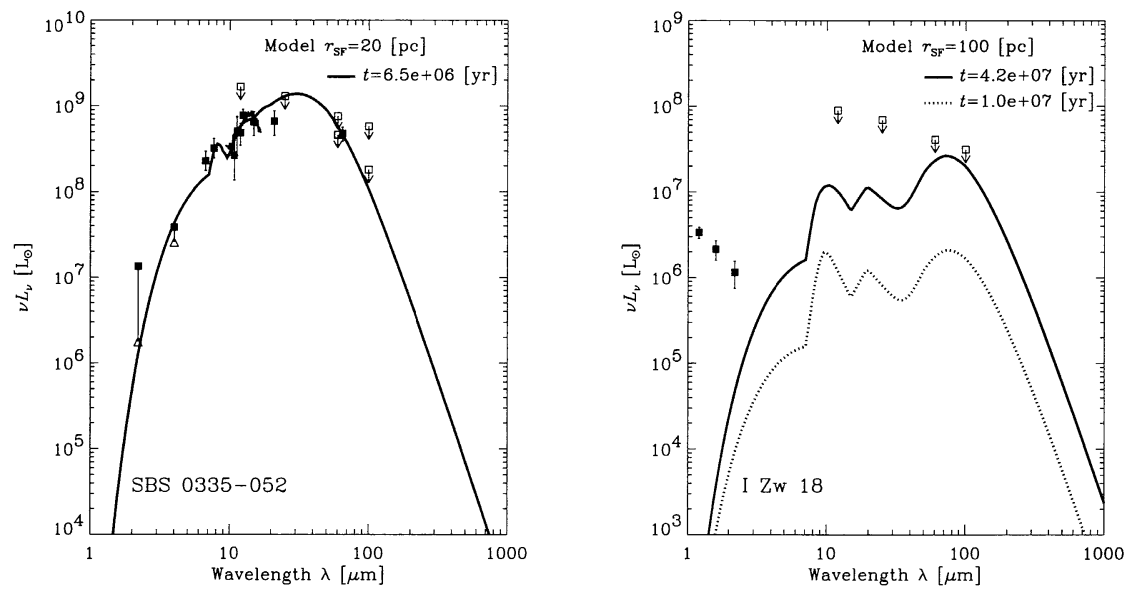

Figure 2. Left: The model SED for SBS 0335-052. Right: The model SED for I Zw 18. 